\title{
Looking for Dark Matter in Neutron Star Light
}

\author{
Radio observations of neutron stars demonstrate a way to search for \\ axions through their expected conversion to electromagnetic waves in a \\ star's magnetic field.
}

By Michael Schirber

S everal laboratory experiments search for axions-a dark matter candidate-using strong magnetic fields to transform axions into photons. Some of the Universe's strongest magnetic fields surround neutron stars, so theorists have proposed also searching for axion signals in neutron star light. Now, a group has done exactly that with two radio telescopes [1]. The lack of a detection allowed the researchers to set world-leading bounds on the light-coupling strength of axions with mass of around $10 \mu \mathrm{eV} / \mathrm{c}^{2}$.

Axion models predict that these hypothetical particles convert into electromagnetic radiation when they are exposed to a strong magnetic field. This possibility has led to a number of experiments using laboratory magnets with strengths approaching ten tesla (see Viewpoint: Homing in on Axions?). Certain neutron stars, by comparison, host magnetic fields as large as a 100 billion tesla. Axion conversions in these enormous fields should produce a sharp peak in emission at a

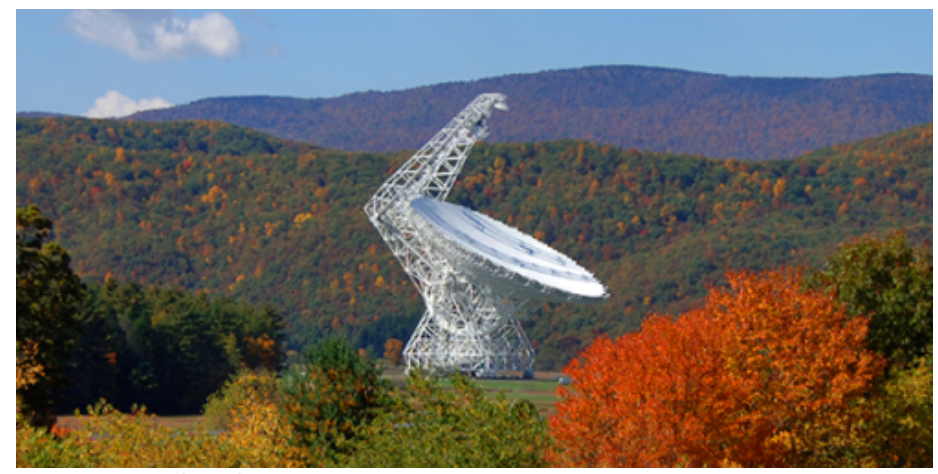

Credit: GBO/AUI/NSF frequency corresponding to the axion mass. However, this signal must contend with similar ones from astronomical and terrestrial sources.

For their axion search, Benjamin Safdi from the University of Michigan and his colleagues performed an analysis of data from the Green Bank telescope in West Virginia and from the Effelsberg telescope in Germany. The targets in this case were two nearby neutron stars known to have strong magnetic fields, as well as the Milky Way's center, which is estimated to host half a billion neutron stars. The team sampled radio frequencies in the 1-GHz range, corresponding to axion masses of 5-11 $\mu \mathrm{eV} / c^{2}$. Although no signal was seen, the team believes that further neutron star observations could become sensitive to very weakly coupled axions, which are predicted in models of the strong nuclear interaction.

Michael Schirber is a Corresponding Editor for Physics based in Lyon, France.

\section{REFERENCES}

1. J. W. Foster et al., "Green Bank and Effelsberg radio telescope searches for axion dark matter conversion in neutron star magnetospheres," Phys. Rev. Lett. 125, 171301 (2020). 